\title{
Reading and social skills programmes had limited effectiveness on behavioural maladjustment in primary school children
}

Hundert J, Boyle MH, Cunningham CE, et al. Helping children adjust-a Tri-Ministry Study: II. Program effects.J Child Psychol Psychiat 1999 Oct;40:1061-73.

QUESTION: In primary school children (up to grade 3), what is the effectiveness of a social skills programme, a partner reading programme, or a combination of the 2 in preventing behavioural maladjustment?

Design

5 year randomised \{allocation concealed*\}, $\uparrow$ unblinded,* controlled trial.

\section{Setting}

Primary divisions of 60 schools in 11 boards of education in Ontario, Canada.

\section{Participants}

\{Children in participating schools who were in the primary grades (mean age $6.5 \mathrm{y}$ ). At the beginning of each school year during the study period, samples of students were randomly selected for follow up, stratified by levels of emotional and behavioural risk. In programme schools about 150 students per school or the entire primary division were sampled. In control schools a $15 \%-20 \%$ random sample of students was selected. $89 \%$ of students were followed up. $\}$ 韦

\section{Intervention}

\{In each year of the study, 1 or 2 schools were randomly selected from each board of education and were randomly allocated to 1 or both programmes (social skills $[\mathrm{SS}]$ and partner reading [RE]). Programme schools maintained their programme status until the end of follow up. Schools that were not selected to implement a programme served as control schools until selected by chance to be assigned to a programme. The programmes were implemented by classroom teachers with coaching from trained facilitators. $\}$ \$

\section{Main outcome measures}

Positive playground behaviour, inappropriate classroom behaviour, reading level, externalising problems rated by teachers and parents, and social skills rated by teachers and parents. Outcomes were assessed using interviewer administered and self completed questionnaires and standard testing in the home, and teacher completed questionnaires and observation in the school.

Sources of funding:

Ontario Ministry of

Education; Ontario

Ministry of Health;

Ontario Ministry of

Community and Social

Services; and 11

Ontario school boards.

For correspondence: $\mathrm{Dr}$

M H Boyle, Centre for

Children at Risk,

McMaster University

Faculty of Health

Sciences and Hamilton

Health Sciences

Corporation, Patterson

Building, Chedoke

Division, Hamilton,

ON L8N 3Z5, Canada

Fax +1905574

6665 .

\section{Main results}

Positive playground behaviour increased in all programme schools to a greater extent than in comparison schools (linear effect was $\beta=5.96 v 1.78$, respectively, $\mathrm{p}<0.05$ ) but did not differ among the 3 programmes. Inappropriate behaviour in the classroom worsened in all schools except for those assigned to SS alone, but none of the growth trajectory slopes were statistically significantly different. Reading improved in all schools including comparison schools with no difference among them. The combination of SS and $\mathrm{RE}$ and SS alone showed modest effects on teacher and parent rated externalising problems $(\mathrm{p}<0.05)$.

\section{Conclusions}

In primary school children (up to grade 3), programmes to enhance social skills and reading to prevent behav- ioural maladjustment were effective in a small number of outcomes. Playground behaviour was improved and externalising problems were somewhat decreased.

*See glossary.

†Information provided by author.

\$Boyle MH, Cunningham CE, Heale J, et al. J Child

Psychol 1999;40:1051-60.

\section{COMMENTARY}

Between $15 \%$ and $20 \%$ of the population have a diagnosable psychiatric disorder. ${ }^{1}$ Treatment could not begin to provide the requisite care, assuming for a moment that effective interventions were available. Prevention provides an opportunity to intervene early to reduce the incidence and ultimately the prevalence rates of a particular problem. The goals of prevention are important and seductive. The challenges are monumental.

The Tri-Ministry Study by Hundert $e t a l$ is exemplary in so many ways including the care with which the interventions were executed, the use of multiple outcome measures, and the sophisticated evaluation of the results. The intervention effects were negligible and did not reveal important changes in the children. The study conveys the key challenges for research and the new directions that are needed.

The research priority is to develop preventive interventions that produce strong and replicable effects. It is premature to compare different interventions. We need to show that any intervention-our most powerful one-can produce replicable and clinically important outcomes. Studies ought to pose mechanisms through which changes may occur, design interventions to change these processes, and assess the relation of these processes to outcome. Until we focus on understanding change, preventive interventions will continue to shoot at the target in darkness. Occasionally, programmes will hit their mark but this will be unreliable. Prevention research, perhaps more than any other area, underscores the importance of the basic-applied research connection. We must better understand how change comes about in order to have reliable effects.

Because preventive interventions are usually large scale, the feasibility of implementing a programme once the investigators leave the setting is a critical issue. If this study yielded powerful effects, could the intervention be implemented elsewhere without the extraordinary talent that this research team represents? This report provides an extremely thoughtful discussion of these and related issues and serves as a guide for others contemplating preventive interventions.

Alan E Kazdin, PhD, ABPP Yale University New Haven, Connecticut, USA

1 Institute of Medicine. Committee for the Study of Research on Child and Adolescent Mental Disorders. Research on children and adolescents with mental, behavioral, and developmental disorders. Washington, DC: National Academy Press, 1989. 